\section{$\underset{\substack{\text { hommes } \\ \text { \& migrations }}}{ }$}

\section{Hommes \& migrations}

Revue française de référence sur les dynamiques

migratoires

$1316 \mid 2017$

L'islam en Europe

\title{
Dialogues entre musulmans et non-musulmans
}

S'apprivoiser pour mieux vivre ensemble en Belgique

\section{Morgane Devries et Altay Manço}

\section{(2) OpenEdition}

1 Journals

\section{Édition électronique}

URL : http://journals.openedition.org/hommesmigrations/3806

DOI : 10.4000/hommesmigrations.3806

ISSN : 2262-3353

\section{Éditeur}

Musée national de l'histoire de l'immigration

\section{Édition imprimée}

Date de publication : 1 mars 2017

Pagination : 119-128

ISBN : 978-2-919040-37-7

ISSN : $1142-852 X$

\section{Référence électronique}

Morgane Devries et Altay Manço, «Dialogues entre musulmans et non-musulmans », Hommes \& migrations [En ligne], 1316 | 2017, mis en ligne le 01 mars 2020, consulté le 20 septembre 2020. URL : http://journals.openedition.org/hommesmigrations/3806; DOI : https://doi.org/10.4000/ hommesmigrations.3806 


\title{
DIALOGUES ENTRE MUSULMANS ET NON- MUSULMANS S'APPRIVOISER POUR MIEUX VIVRE ENSEMBLE EN BELGIQUE
}

Par MORGANE DEVRIES, collaboratrice de l'Irfam, et ALTAY MANÇO, directeur scientifique de l'Irfam.

\author{
Les représentations négatives et les préjugés culturels dont \\ sont victimes les populations immigrées, en particulier musul- \\ manes, questionnent les modalités de la compréhension de \\ I'autre. Cet article présente différentes approches et méthode \\ pour mettre en œuvre des dialogues entre musulmans et non- \\ musulmans. Les approches institutionnelle, pédagogique et \\ confessionnelle concourent à poser les bases d'une compré- \\ hension entre les communautés, afin de leur permettre de \\ construire ensemble leur avenir.
}

À l'heure où le religieux et l'islam questionnent les habitants européens, autant du côté des communautés musulmanes que non musulmanes, il importe d'analyser la manière dont ces derniers perçoivent les valeurs défendues par l'Europe, en vue de faire disparaître les crispations qui empêchent le dialogue. Une enquête commanditée par l'Union européenne (UE) et réalisée dans dix pays membres sur le rapport entre musulmans et non-musulmans met en exergue la convergence des acteurs sur les valeurs de la liberté d'expression, le respect de la loi et l'égalité des chances. La seule divergence qui est mentionnée porte sur le respect des religions, valeur qui semble plus importante pour les personnes de confession musulmane ${ }^{1}$. Ce constat met en lumière l'actuelle interrogation sur l'adéquation entre démocratie et religion, rendue notamment visible par l'islam au travers de certaines pratiques dans la sphère publique et, plus particulièrement, par la question de la radicalisation ${ }^{2}$.

Aussi, face à des polarisations vis-à-vis de la place qui devrait être donnée à l'islam en Belgique - deuxième religion du pays ${ }^{3}$-, la question du « vivre-ensemble » devient une préoccupation urgente, tant pour les politiques publiques que 
pour la société civile soucieuse d'un meilleur devenir de nos sociétés démocratiques. Le « vivreensemble»se définit parla construction d'ententes réciproques, pacifiques et respectueuses des personnes dans leurs identités culturelles et religieuses, en considérant la

Ainsi, en l'absence d'une autorité spirituelle unifiée, la question de la représentation

et de la gestion de l'islam en Belgique semble délicate

tant les populations et

les croyances concernées

peuvent être diverses,

d'autant que ces politiques régulatrices s'inscrivent dans des débats sociétaux et une

ambiance médiatique et sociale marquée par la peur de l'islam. reconnaissance des diversités comme fondement de la cohésion sociale d'une société démocratique 4 . Cet article vise ainsi à répertorier différentes démarches soutenues en Belgique dont les apports visent, directement et indirectement, à valoriser un dialogue entre musulmans et non-musulmans. Ces approches $^{5}$ correspondent à des niveaux différents - institutionnel, pédagogique et d'obédience - et sont analysées ici par le biais de points de repère transversaux : leur contexte d'émergence, les enjeux qu'elles soulèvent, les acteurs mobilisés et les méthodes qu'elles requièrent ou à travers lesquelles elles sont appréhendées. Le but de cette présentation est de montrer leur complémentarité et singularité dans leur dynamique favorable au « vivre-ensemble».

\section{Les approches juridico-légales et institutionnelles}

Ces approches visent la production et/ou l'usage de normes, de lois et de réglementations afin de définir un support structurel à une inclusion de l'islam au sein de la société : soit à travers des organes institutionnels qui en garantissent la représentation, soit au sein d'institutions qui doivent se positionner par rapport à l'expression du religieux en leur sein. Elles s'inscrivent autant dans le champ institutionnel des luttes contre les discriminations que dans celui de la liberté de culte, et sont considérées comme des cadres référentiels dans la gestion des cultes et de leur expression publique.

En Belgique, l'intervention de l'État dans le temporel des cultes est fondée sur des habitudes séculières à l'égard les religions majoritaires. Au XXe siècle, dans un contexte général de sécularisation, la lente construction de parallélismes entre les façons de traiter les religions majoritaires et les religions (très) minoritaires, ainsi que les spiritualités non religieuses ne semble pas avoir posé beaucoup de difficultés. La constitution belge, à ce titre, reconnaît les régimes des cultes et des convictions philosophiques (art. 19), garantie la liberté de conscience (art. 20) et les principes de pluralisme et de non-discrimination (art. 11). Cela étant, la littérature sur ce sujet en Belgique relève de nombreux problèmes : inadéquation des espaces de prière et prières collectives dans la rue, difficile incorporation urbanistique des mosquées dans les villes, questions de la formation et de la professionnalisation des imams et des enseignants de la religion, mais aussi dans la production et la commercialisation de la viande halal, etc ${ }^{6}$. La question épineuse de l'articulation entre le religieux et l'espace public ne peut donc, par cette seule reconnaissance institutionnelle, trouver une réponse $e^{7}$. Ainsi, en l'absence d'une autorité spirituelle unifiée, la question de la représentation et de la gestion de l'islam en Belgique semble délicate tant les populations et les croyances concernées

\footnotetext{
4. Le « vivre-ensemble " n'exclut pas l'existence de rapports de force ou de conflits. Au contraire, il incorpore le débat contradictoire comme fondement du changement social et du processus de reconnaissance réciproque entre citoyens. 5. Notre démarche n'a pas vocation à être exhaustive, mais tend à valoriser les différentes modalités de dialogue présentes en Belgique. 6. Elena Arigita, "Representing islam in Spain: Muslim identities and the contestation of leadership ", in The Muslim World, vol. 96, n 4, 2006 ; Jean-François Husson, La formation des imams en Europe. État des lieux, Bruxelles, Fondation Roi Baudouin, 2007. 7. Voir au sujet d'un historique du rapport entre la législation belge et de son rapport au religieux: Xavier Delgrange, Hélène Lerouxel, "L'accommodement raisonnable, bouc émissaire d'une laïcité inhibitrice en Belgique ", in Emmanuelle Bribosia, Isabelle Rorive (dir.), L'accommodement de la diversité religieuse. Regards croisés Canada, Europe, Bruxelles, Peter Lang, 2015, pp. 203-286; Ilke Adam, Andrea Rea, "Les pratiques d'accommodements raisonnables sur les lieux du travail en Belgique ", in Emmanuelle Bribosia, Isabelle Rorive (dir.), op. cit., pp. 313-343.
} 
peuvent être diverses, d'autant que ces politiques régulatrices s'inscrivent dans des débats sociétaux et une ambiance médiatique et sociale marquée par la peur de l'islam.

À travers ce système, l'État belge garantit, pour tout culte et sous couvert du respect de certaines conditions ${ }^{8}$, d'engager un processus de reconnaissance nécessaire à l'octroi de subsides' ${ }^{9}$. Par conséquent, les enjeux de ces approches se concentrent sur les possibilités d'un dialogue entre l'État et l'autorité du culte et concernent la représentativité et le leadership musulman. Cette question est débattue, revue et corrigée au gré d'enjeux géopolitiques, et elle tarde à mettre en cohérence les attentes des communautés musulmanes, en termes d'autorité religieuse et de pratique cultuelle, avec celles de l'État belge pour un islam contextualisé, et les réalités institutionnelles belges en termes de neutralité. Cette réglementation des pratiques islamiques implique un arsenal juridique et des appareils institutionnels ${ }^{10}$, dont la constitution belge, qui intègrent de diverses manières des processus de consultation et de négociation avec des groupes musulmans ou non, immigrés ou non ${ }^{11}$. Ainsi, les méthodes qui prévalent au sein de ces approches sont généralement tributaires du cadre législatif et de la capacité des communautés musulmanes à organiser leur culte ${ }^{12}$, mais aussi de la volonté du pouvoir politique d'affirmer son rôle de coordinateur. De facto, ces approches visent ainsi à créer un pont favorable au dialogue là où, en amont, il n'y en avait pas ou peu.

\section{Les approches socio-éducatives pour le dialogue interculturel}

Ces approches visent la construction d'actions nationales ou locales œuvrant à une co-inclusion de l'islam par le biais d'une démarche pédagogique d'apprentissage, d'interconnaissance et de compréhension, en vue de déconstruire les préjugés mutuels, et ainsi de lutter contre les discriminations et de développer une cohésion sociale. En outre, elles contribuent à un dialogue réciproque entre musulmans et non-musulmans à travers la valorisation d'une appartenance citoyenne commune. En raison d'une sécularisation pluraliste ${ }^{13}$, ce qui a été perçu comme un "retour du religieux ${ }^{14}$ » nous rappelle que ce dernier, bien que moins institué, n'a pas fait disparaître le besoin de spiritualité. Vers le milieu des années 1970, à travers la visibilité croissante des populations musulmanes ${ }^{15}$ et leur demande accrue pour l'exprimer publiquement, l'islam n'a eu de cesse d'alerter les municipalités locales, les acteurs associatifs et professionnels sur la gestion des rapports sociaux face à l'impact du terrorisme et de l'actualité internationale sur l'image des musulmans. L'approche socioéducative a eu cette particularité de s'amorcer à la demande des acteurs (commanditaires publics ou sociaux) désireux de lutter contre les discriminations tout en promouvant une démarche participative des communautés.

\footnotetext{
8. Parmi d'autres nombreuses conditions : un culte peut être reconnu une fois qu'il est présent depuis dix ans sur le territoire belge et si les membres sont suffisamment nombreux. Voir Jean-François Husson, "Le financement public de l'islam - instrument d'une politique publique? ", in Benedicte Maréchal, Farid El Asri (dir.), Islam belge au pluriel, Louvain-la-Neuve, Presses universitaires de Louvain, 2012, pp. 241-258. 9. Les subsides de l'État sont consacrés aux éléments qui suivent : les frais de culte, les bâtiments et leur traitement, les aumôneries et une émission de télévision concédée. Voir Ibid. 10. Nous aurions pu évoquer les "Plans de diversité » ou autres lois/règlements visant à réguler et lutter contre les discriminations directes et indirectes notamment, mais pas seulement, en matière de critère convictionnel : loi Moureau de 1981 contre le racisme, la création d'Unia (Centre interfédéral pour l'égalité des chances) en 1993, la réforme de la loi anti-discrimination de 2007 visant à transposer les directives de l'UE. Voir Dounia Bouzar, Nathalie Denies, Diversité convictionnelle. Comment l'appréhender? Comment la gérer ?, Louvain-la-Neuve, L'Harmattan, 2014. 11. Thijl Sunier, « Domesticating Islam: Exploring Academic Knowledge Production on Islam and Muslims in European Societies ", in Ethnic and Racial Studies, vol. 37, $n^{\circ}$ 6, 2014. 12. Louis-Léon Christians, "Sustainable Management of Religious Diversity: An Overview of the Belgian Model and its Prospects for Success ", in MarieClaude Foblets, Jean-François Gaudreault-DesBiens, Alice Dundes Renteln (dir.), Cultural Diversity and the Law. State Responses from Around the World, Montréal/Bruxelles, Yvon Blais/Bruylant, 2010, pp. 819-843. 13. Yves Lambert, « Le rôle dévolu à la religion par les Européens ", in Sociétés contemporaines, $n^{\circ}$ 37, 2000, p. 32. 14. Marcel Gauchet, Le désenchantement du monde. Une histoire politique de la religion, Paris, Gallimard, 1985. 15. Felice Dassetto, La construction de l'Islam européen : approche socioanthropologique, Paris, L'Harmattan, 1996 ; Felice Dassetto, «L'islam en Belgique et en Europe : facettes et questions ", in Felice Dassetto (dir.), Les facettes de l'islam belge, Louvain-la-Neuve, Académie-Bruyant, 1997, pp. 17-34; Jocelyne Cesari, «L'islam en Europe ", in Cahiers d'études sur la Méditerranée orientale et du monde turco-iranien, vol. 33, 2002.
} 
Comme l'évoque Bosset ${ }^{16}$, au sein des sociétés occidentales, nous avons affaire à des "dialogues ", parfois bilatéraux, souvent multiformes, parmi lesquels certaines convergences de pratiques sont observables et concourent à mieux gérer la diversité culturelle et religieuse, en complément et en amont de la voie juridique et normative. Ce dialogue vise donc principalement la prévention de conflits par une approche compréhensive, consultative et de relais d'informations relatives à la cohabitation du religieux. Il complète les apports du système juridique et institutionnel par un travail sur les représentations. En termes d'enjeux, il s'agit d'impulser une dynamique de long terme dont la portée concerne des groupes à un niveau micro ou méso, dépendant de la diffusion et de la transmission de leurs résultats entre acteurs concernés.

La méthode privilégiée est essentiellement celle de la recherche-action, dont le résultat prend des formes multiples : formations, médiations, actions de sensibilisation, espaces de débats et de consultation ou publication de recommandations. Elle se déroule en plu-
La méthode privilégiée est essentiellement celle de la recherche-action, dont le résultat prend des formes multiples formations, médiations, actions de sensibilisation, espaces de débats et de consultation ou publication de recommandations. sieurs phases $^{17}$ : une phase d'identification du problème et des situations au sein desquelles prend part le projet, l'établissement d'hypothèses (propositions de réponses possibles), l'élaboration d'un plan d'action, son déroulement et, enfin, son évaluatio $\mathrm{n}$ à partir des résultats analysés. L'approche invite les citoyens à collaborer à un projet avec d'autres professionnels à des fins diverses : consultation, expression et partage d'expériences, délibération, etc. À cet égard, les actions menées par le biais de cette approche ont pour spécificité de s'intégrer et/ou de promouvoir un travail en réseau. Ces collaborations associent généralement des acteurs associatifs et des professionnels (enseignants, travailleurs sociaux, éducateurs, etc.), ainsi que, dans une moindre mesure, des représentants politiques locaux et des citoyens. Par conséquent, l'engagement de citoyens au processus participatif de la recherche se double d'une co-construction de savoirs des chercheurs dans une perspective de changement social.

\section{Les approches théologiques pour le dialogue interreligieux}

Ces démarches visent des actions nationales ou locales qui œuvrent à une cohabitation des diversités convictionnelles et/ou à l'examen de ces diversités. D'une part, une démarche intracommunautaire se concrétise par une réflexion théologique (dogmes, doctrines, textes sacrés, etc.) en vue de faciliter des comparaisons entre religions, des contextualisations et adaptations par interprétation religieuse. D'autre part, une démarche intercommunautaire, à travers des rencontres entre communautés, invite les participants à partager leur fondement (croyances, pratiques, rites, etc.) dans la société civile. Au sein de la société sécularisée, le croire et la religiosité semblent s'individualiser ${ }^{18}$. Cela étant, l'ancrage communautaire et le leadership religieux restent un socle de sociabilité et d'identification des interlocuteurs au service du « vivre-ensemble » entre les différentes religions. Au sein de sociétés pluralistes, les rencontres multiconfessionnelles ou interreligieuses ont pour point d'orgue la réconciliation, de sorte que les communautés religieuses puissent cohabiter et apporter leur participation collective au dialogue, malgré les crispations identitaires ${ }^{19}$.

Ce type d'approche se base sur un dialogue en vue de bâtir la paix, de manière générale de privilégier un message spirituel commun à travers les messages délivrés par les diverses religions malgré leurs antagonismes. Les défis sont aujourd'hui liés à la mon- 
tée de l'islamophobie, de l'antisémitisme, de la xénophobie et des sentiments antichrétiens susceptibles de mettre à mal les particularismes culturels et religieux des identités individuelles. Pour reprendre les mots du président belge du Conseil pontifical pour le dialogue interreligieux, lobjectif est de " passer de la peur à la confiance ${ }^{20}$ ».

Nous retrouvons également une dynamique de projets multiconfessionnels au sein de la société civile belge, sous des formes variables comme des semaines islamo-chrétiennes, des parcours-découvertes au sein de lieux de culte, des concerts religieux ou des festivals, des conférences ou des séminaires, des repas collectifs ${ }^{21}$ et des formations ${ }^{22}$. La démarche privilégie un examen théologique de la part des leaders religieux. Les acteurs sont les municipalités, les chefs de culte et les institutions religieuses qu'ils représentent (églises, mosquées, synagogues, etc.), des théologiens, les croyants des religions représentées, et diverses organisations et associations qui proposent des activités menées dans la citée ${ }^{23}$.

\section{L'institutionnalisation de l'islam}

Comme le souligne Ural Manço $\mathrm{O}^{24}$, le processus de reconnaissance ou d'institutionnalisation de l'islam en Europe est particulièrement sensible aux réalités nationales et à l'actualité internationale. Cette question n'est d'ailleurs toujours pas entièrement résolue dans de nombreux pays du fait également de l'influence des pays d'origine ou de pays tiers musulmans ${ }^{25}$. Mais la demande d'avoir un représentant pour le culte islamique est un impératif de l'État belge. Selon Jonathan Laurence, plusieurs pays d'Europe occidentale ont cherché, durant les années 1990, à identifier dans la société civile auprès d'associations de migrants musulmans des interlocuteurs " modérés ", « légitimes » et « représentatifs » pour envisager la possibilité de les organiser en organe de gestion de culte $^{26}$. L'objectif était d'entrevoir une bonne coordination entre l'appareil de l'État et les responsables de cet organe, en échange du monopole sur le dogme religieux. La littérature laisse apparaittre deux manières de construire la représentation de l'islam en Europe ${ }^{27}$ : soit par les négociations et les pondérations d'une part, soit par les élections d'autre part, bien qu'elles puissent se combiner.

En Belgique, un groupe d'étudiants musulmans et de réfugiés albanais a été sollicité au début des années 1960 par des ressortissants ou des représentants de pays musulmans (Tunisie, Arabie saoudite et Maroc) afin de les aider à constituer un lieu de culte à Bruxelles. Cette association a été reconnue par l'État belge comme le Centre culturel et islamique en 1968. S'amorce alors un climat favorable qui a conduit à la reconnaissance ultérieure du culte islamique par la constitution belge en 1974 dans un contexte commercial à l'avantage des pays du Golfe. Par la suite, le processus de reconnaissance du culte musulman a connu de très nombreux obstacles ${ }^{28}$ si bien qu'il reste toujours inachevé à ce jour. Aujourd'hui, la relation qui unit cet organe organisateur du culte musulman et l'État belge s'avère plus qu'ambiguë, au regard de la neutralité tant valorisée par les discours actuels et du principe laïque de la séparation entre l'État et la religion, d'autant plus que les autres cultes ou philosophies reconnus sont proportionnellement mieux financés et moins encadrés que le culte musulman.

20. Jean-Jacques Durré, "Dialogue interreligieux : passer de la peur à la confiance », in Cathobel, 30 octobre 2015 [en ligne]. 21. Le 17 décembre 2016, par exemple, un dîner interreligieux de Noël s'est déroulé à Molenbeek. Voir Nicolas Senèze, "Le dialogue des communautés religieuses, l'autre visage de Molenbeek ", in La Croix, 22 décembre 2015 [en ligne]. 22. Le groupe "Coexister " est le mouvement interreligieux des jeunes - présent aussi en France - qui propose des formations afin de transmettre les fondements des religions à d'autres personnes croyantes ou non croyantes. 23. Groupe de rencontres et d'actions interreligieuses (Grair), Aisa Belgique, Ensemble pour la paix et la fraternité, Les amis de l'islam, Centre El Kalima, Groupe Taizé Flagey, Pax Christi, Bruxelles vivreensemble, etc. 24. Ural Manço (dir.), Reconnaissance et discrimination. Présence de l'islam en Europe occidentale et en Amérique du Nord, Paris, L'Harmattan, 2004. 25. John R. Bowen, Why the French Don't Like Headscarves: Islam, the State, and Public Space, Princeton, Princeton University Press, 2007, pp. 11-63. 26. Jonathan Laurence, The Emancipation of Europe's Muslims: The State's Role in Minority Integration, Princeton, Princeton University Press, 2012, p. 7. 27. Jocelyne Cesari, L'Islam à l'épreuve de l'Occident, Paris, La Découverte, 2004. 28. Johan Leman, " Minority leadership, science, symbols and the media: The belgian islam debate and its relevance for other countries in Europe ", in Journal of International Migration and Integration, vol. 1, n 3, 2000, pp. 351-372. 


\section{La formation des cadres musulmans}

Dès 1978, l'État a modifié la Loi du Pacte scolaire et y a introduit les professeurs de religion islamique et la religion islamique parmi les cours philosophiques, en application de la loi du 19 juillet 1974 reconnaissant le culte islamique. Or, l'islam n'est pas doté à ce jour d'institutions religieuses et d'encadrement ou d'espaces de débat dédiés comme c'est le cas pour d'autres confessions. Par ailleurs, il ne dispose pas non plus d'une émission sur une chaine nationale comme devrait la lui garantir la constitution. Face aux relations étroites entre les mosquées et les États nationaux musulmans, promouvoir un islam " de Belgique " passe par la création d'un réseau dense de formations et de recherches scientifiques de qualité sur l'islam et sur le monde musulman, notamment à destination des jeunes générations. Il s'agit d'offrir un socle de connaissances destinées aux
Dès 1978, l'État a modifié la Loi du Pacte scolaire et $y$ a introduit les professeurs de religion islamique et la religion islamique parmi les cours philosophiques, en application de la loi du 19 juillet 1974 reconnaissant le culte islamique. professeurs de religion islamique et aux imams, tous deux non soumis, pour l'instant, à des critères spécifiques. C'est pourquoi, depuis 2013, le cabinet Marcourt ${ }^{29}$ a demandé un rapport pour réfléchir à la formation de cadres musulmans, de professeurs de religion islamique, de conseillers moraux en milieu pénitentiaire et hospitalier, de médiateurs culturels, etc. Face à ce défi, Édouard Delruelle ${ }^{30}$, conseiller du ministre Marcourt, nous informe qu'une commission a été créée en mars 2015 en vue de faire émerger un islam de Belgique en Fédération Wallonie-Bruxelles, en col- laboration avec l'Exécutif musulman de Belgique. Elle est dirigée par Andrea Rea ${ }^{31}$ et Françoise Tulkens $^{32}$. Le 4 décembre 2015, son rapport ${ }^{33}$ formule des propositions, parmi lesquelles figure en première place la création d'un institut de formation et de profession de l'islam et l'organisation d'une émission concédée au culte musulman. Au cours de l'année 2016, le cabinet a avant tout travaillé à la création de l'institut en question. Celui-ci n'aura pas pour vocation d'organiser lui-même les formations et donc à diplômer - cela reste le rôle des écoles - ; son rôle sera de promouvoir, de soutenir et de financer, le cas échéant, des formations et des recherches concernant l'islam à destination des publics évoqués ci-devant. Rea et Tulkens coordonneront l'établissement de cette institution. La création et l'organisation d'une chaire visant à favoriser la formation critique de la pensée arabo-musulmane dans ses dimensions contemporaines ne sont pas envisagées. L'institut vient donc s'ajouter à des initiatives en matière de formations d'ores et déjà prises par des universités ${ }^{34}$.

\section{Échanges de « bonnes pratiques » de dialogues locaux}

L'échange de "bonnes pratiques » est un procédé de partage et de mise en débat qui se situe à un niveau supra local des modes de gouvernance et de gestion des diversités culturelles entreprises aux échelles locales. Le Programme commun pour l'intégration des ressortissants de pays tiers dans l'Union européenne valorise la place des initiatives de coordination et d'échange de pratiques d'intégration au niveau national et local. Les études ou les recherches-actions établissent un bilan comparé dans une démarche d'évaluation transversale entre pays, régions et localités. L’analyse des initia-

29. Jean-Claude Marcourt est, entre autres, le ministre-président de l'Enseignement supérieur de la Fédération WallonieBruxelles depuis 2009, et est ministre de la Recherche et des médias depuis 2014. 30. Discours d'inauguration de la nouvelle "Chaire Islam : histoire, cultures et sociétés », Université libre de Bruxelles (27 octobre 2016). 31. Andrea Rea est professeur de sociologie à l'université libre de Bruxelles et membre du Groupe pour les études sur les relations ethniques et l'égalité (Germe). 32. Françoise Tulkens est professeure émérite à l'université catholique de Louvain et ancienne vice-présidente de la Cour européenne de justice. 33. www.uclouvain.be/cps/ucl/doc/cismoc/documents/Rapport_final_commission_Marcourt(1). pdf. 34. Pour un aperçu des autres formations d'ores et déjà mises en œuvre par les universités belges relatives à l'islam, voir l'article de S. Grawez : www.uclouvain.be/cps/ucl/doc/cismoc/documents/Belgiciser.pdf 
tives développées par des politiques d'intégration nationales est un moyen par lequel des recommandations peuvent être partagées entre diverses entités de l'UE.

Laction «Cultes et cohésion sociale » a vu le jour dans le cadre du Programme européen de lutte contre la discrimination et pour les droits sociaux fondamentaux et la société civile (art. 13 du traité de l'Union). Il s'agit d'une action d'identification, de validation et d'échanges transnationaux de «bonnes pratiques » et d'informations dans le domaine de la lutte contre les discriminations coordonnée par l'Institut de recherche, formation et action sur les migrations de Liège ${ }^{35}$. Elle est menée sur un plan transnational avec six autres pays européens (France, Espagne, Italie, Belgique, Grande-Bretagne), ainsi que le Québec et le Massachusetts. Le diagnostic mené au départ de l'initiative ${ }^{36}$ montre que la religion musulmane s'est implantée de manière visible dans les sociétés. Pourtant, de nombreux musulmans européens peinent à se faire reconnaître dans leur identité religieuse et à exprimer leurs pratiques islamiques dans l'espace public malgré leur attachement à cette reconnaissance. En étudiant les effets de cette reconnaissance, la recherche-action débouche sur des observations locales à propos de la prise en compte publique des identités et des demandes musulmanes ${ }^{37}$ : de quelles manières les administrations locales gèrent la diversité religieuse et, en particulier, la présence musulmane et ses infrastructures, comme les mosquées?

Ainsi, le réseau "Cultes et cohésion sociale » a réuni de 2001 à 2005 un ensemble de partenaires (chercheurs, acteurs/décideurs locaux et représentants des populations locales) pour une série de rencontres nationales et transnationales dans une dizaine de villes européennes, et a produit un guide de «bonnes pratiques » adoptées par les administrations municipales favorisant un dialogue avec les communautés musulmanes. Selon Manço et
Amoranitis, "laction et l'observation locales permettent d'atteindre un savoir pratique sur les modalités les plus efficaces d'une intervention visant à prévenir et à dépasser des situations de blocage38 ». Elles sont influencées par des dynamiques locales et leurs résultats sont variables selon les contextes d'émergence. Ce différentiel représente un enjeu pour la compréhension et la diffusion des "savoirfaire " de négociation entre communautés et administrations. L'intérêt repose sur la possibilité donnée aux décideurs et aux acteurs sociaux européens de s'approprier ces méthodes, à leur manière et en tenant compte de leurs réalités locales, et d'œuvrer
L'intérêt repose sur la possibilité donnée aux décideurs et aux acteurs sociaux européens de s'approprier ces méthodes, à leur manière et en tenant compte de leurs réalités locales, et d'œuvrer avec créativité à l'évolution et à la pérennisation de celles-ci. avec créativité à l'évolution et à la pérennisation de celles-ci. Un exemple en serait la création d'espaces de confrontation favorisant "la reconnaissance sociale et l'accès à la citoyenneté de tous ${ }^{39}$ ". Les échanges de «bonnes pratiques » et l'évaluation des méthodes répertoriées identifient ainsi les trames communes aux différentes expériences locales.

\section{Les forums réflexifs}

Les forums favorisent les échanges de points de vue entre individus ou groupes " appartenant à des univers culturels différents, permettant à chacun de mieux comprendre la vision du monde de l'Autre et favorisant, ainsi, la coexistence pacifique des différents groupes ethnoculturels au sein d'une sociététe ". Composant une bonne partie des pratiques présentées par Manço et Amoranitis ${ }^{41}$ et Haddad, Manço et Eckman ${ }^{42}$, les « forums réflexifs » sont également développés par de Changy, Dassetto et Maréchal ${ }^{43}$ 
et Amahjour, Della Piana et Herman ${ }^{44}$. Autant le travail de Manço et Amoranitis ${ }^{45}$ part de " problèmes " précis (la demande de construction d'une mosquée, d'un cimetière musulman, etc.), et met en situation de négociation des municipalités et des associations d'habitants (musulmans ou non), à travers l'Europe, autant l'initiative de de Changy et al. ${ }^{46}$ cherche plutôt à cerner les tensions générales présentes dans les relations entre citoyens musulmans et non-musulmans dans plusieurs villes belges. Celle de Amahjour et al. ${ }^{47}$ soulève des interrogations en interaction. Dans les deux premiers cas, ces forums sont des moments de débats à l'occasion desquels des personnes différentes peuvent échanger leur avis sur des questions sensibles relatives aux rapports entre musulmans et non-musulmans (valeurs morales, espace public/ privé, rapports entre hommes et femmes, jeunes et aînés, interdits alimentaires, temps et d'espaces partagés, etc.). En ce sens, l'outil prend en compte la dimension religieuse du vivre-ensemble en y dégageant les argumentations des uns et des autres, en particulier vis-à-vis de l'islam.

Dans ce premier cas, le contact entre décideurs et responsables de la société civile est assuré par la présence d'experts et est
À la suite d'un premier cycle de recherche-formation «Europe et Islam : que avenir ? Un chantier à mener ensemble » à Bruxelles, à Ottignies, à Charleroi et à Liège, sont apparues des peurs relatives à un islam « envahissant ». orienté vers la résolution de problèmes locaux concrets (approche interventionniste). Dans le second, des citoyens participent à un échange organisé par les chercheurs dans le but d'identifier « les signaux de malaise et de conflictualité qui ont surgi et s'amplifient du fait de la présence nouvelle de l'islam en Belgique, sans les réduire à cette cause unique $^{48} »$. Enfin, la recherche-action menée par le centre de formation Cardijn et sagesse au quotidien ${ }^{49}$ témoigne également de la philosophie des forums réflexifs, par le biais de groupe d'échanges au sein d'un cycle de formations intitulé "Regards croisés sur nos questions de sens ». À la suite d'un premier cycle de recherche-formation « Europe et Islam : quel avenir ? Un chantier à mener ensemble » à Bruxelles, à Ottignies, à Charleroi et à Liège, sont apparues des peurs relatives à un islam " envahissant ». Aussi, l'ensemble de ces forums ne se destine pas à occulter la conflictualité et la question des convictions. Porteurs de changement social, ils tentent de décloisonner les postures défensives du "vous/nous » autour de la citoyenneté. Contrairement aux deux exemples précédents, l'expérience la plus récente a déplacé la focale de la dimension religieuse - sans pour autant éluder le rapport aux convictions - sur des sujets convergents et des questions sociales communes aux participants musulmans, chrétiens et non-croyants, à partir du sens sous-jacent qu'ils leur donnent.

\section{Le mode d'emploi de ces forums}

Ces forums réflexifs visent à mettre en évidence des points d'accord et de désaccord et à déceler des signes de confiance, de méfiance ou de défiance entre citoyens musulmans et non musulmans. Ils donnent à des personnes ayant des avis divergents l'opportunité de débattre d'un phénomène problématique qui interpelle tout le monde, et permettent de dégager les enjeux sensibles, les incompréhensions ou les points de tension. Ces forums donnent aux membres de la société un rôle d'acteur et créent des lieux de rencontre entre citoyens pour faire émerger un terrain d'entente à propos du vivre-ensemble, faire se confronter des individus, mais aussi des systèmes de pensée, et permettre aux participants d'acquérir de nouvelles connaissances ou sensibilités et de tendre vers des solutions communes aux problèmes soulevés. Mettre en place de tels forums nécessite de réflé- 
chir au thème au centre de l'enjeu qui doit être unificateur et mobilisateur (étape 1), à la composition des groupes qui doit favoriser le débat et le changement social (étape 2), aux lieux et dates, aux processus de négociation au long cours, à leur animation, bref à la mise en œuvre concrète du dialogue (étape 3), ainsi quà son évaluation et à sa poursuite, par exemple par une démarche d'écriture et de diffusion ou de mise en pratique des recommandations produites en commun (étape 4).

Une attention particulière doit être portée à la représentativité des groupes pour se rapprocher d'un idéal de pluralisme. Si l'objectif est de dépasser un conflit, un problème collectif, la participation conjointe d'acteurs doit jeter des ponts entre les «savoirs d'expérience » et le "savoir savant » dans la construction d'une pensée qui se veut réflexive. Enfin, le public en débat - qui peut évoluer dans le temps - doit être suffisamment hétérogène pour refléter au mieux les diversités internes aux groupes en contact (âge, genre, conditions socio-économiques, convictions, etc).

Selon de Changy et $a .^{50}$ et Haddad et al. ${ }^{51}$, certaines conditions sont favorables à l'émergence et à la résolution de débats entre témoins aux trajectoires personnelles et spirituelles différentes : un cadre de confiance, égalitaire et respectueux de la parole de tous; l'accord préalable sur un thème légitime pour les uns et les autres; un rythme soutenu de rencontres (deux fois par semaine); une animation, une analyse et un suivi centrés sur les échanges (par une personne ou une équipe tierce reconnue par les parties) qui tablent tant sur les différences de point de vue que sur les similitudes; une préparation des rencontres par les parties ou entre les parties, le cas échéant par un processus de médiation, etc. En reconnaissant une capacité de réflexion aux acteurs, cette méthode promeut le pouvoir d'agir à travers des prises paroles. La communication est une forme d'action sociale ${ }^{52}$ qui a un effet sur l'interconnaissance entre parties prenantes. Elle produit de l'empathie réciproque et fait rayonner cette compréhension au-delà du groupe des participants. Elle ouvre alors vers des possibilités d'initiatives collectives, sur le terrain, visant une solution équitable aux problématiques envisagées.

Un autre effet notable des forums réflexifs est de situer le débat entre personnes aux sensibilités et aux attentes différentes dans le champ de la citoyenneté et du vivre-ensemble et non de la légitimité ou non d'une posture religieuse. Selon Ama- L'analyse des actions menées hjour, Della Piana et Her$\operatorname{man}^{53}$, la méthodologie fondée sur des récits et des témoignages d'expériences permet de favoriser l'universalité des valeurs et de les croiser, ultérieurement, à des questions de transmission et de spiritualités. Aussi, elles contribuent à faire de ces démarches une expérimentation, laissant en Belgique démontre que l'importance des logiques légales et institutionnelles ne suffit pas à amoindrir le poids des représentations dans l'appréhension que les populations peuvent avoir de l'expression et des demandes religieuses émanant des communautés musulmanes dans un contexte de tensions identitaires place à l'informalité et à une souplesse d'adaptation. Elles mettent l'accent sur une période de longue durée et, finalement, elles partent d'un thème issu d'un monde commun afin de voir comment celui-ci est appréhendé par des univers confessionnels ou non pour revenir, ensuite, sur une vision projective de sens.

\section{La participation citoyenne, une démarche de dialogue à promouvoir}

L'analyse des actions menées en Belgique démontre que l'importance des logiques légales et institutionnelles ne suffit pas à amoindrir le poids des représentations dans l'appréhension que les populations peuvent avoir de l'expression et des demandes religieuses émanant des com- 
munautés musulmanes dans un contexte de tensions identitaires ${ }^{54}$. D'autre part, comme le met en exergue la recherche de Bocquet, Maréchal et Van Den Abbeele ${ }^{55}$, le changement pour un meilleur « vivre-ensemble » entre musulmans et nonmusulmans nécessite un acteur central en filigrane des politiques menées, à savoir le milieu associatif et ses actions collectives au niveau local. La souplesse, comme la complexité, du cadre législatif belge permet l'existence d'approches socio-éducatives locales. Ces dernières permettent, à leur tour, une appropriation citoyenne de la composante religieuse, à travers des réponses informelles, accueillantes et compréhensives. Le leitmotiv de ces démarches reste l'importance de l'expérimentation et de l'engagement citoyen qui, bien que moins médiatisées et structurelles, sont des laboratoires de négociations et d'échanges sur la présence de l'islam dans l'espace public. De plus, ces démarches participatives ${ }^{56}$ construisent, en partie, leur validité grâce à la présence d'acteurs concer- nés qui vivent au quotidien dans la diversité, la coconstruction et la réciprocité entre communautés. Dans ces processus, des acteurs locaux sont formés et légitimés à débattre et à apaiser des tensions. C'est un avantage pour prévenir les conflits liés, la plupart du temps, à des incompréhensions ou des méconnaissances mutuelles. Il s'agit de mutualiser et de partager ces initiatives à de plus larges échelles, notamment en Belgique, où le système politique tend à laisser l'interprétation et les décisions des législations en matière de culte aux institutions locales. Cela laisse la place aussi, paradoxalement, à des rapports de force et à une gestion inégalitaire ${ }^{57}$ entre acteurs qui pourraient trouver, dans ces approches, des pratiques efficaces pour leurs besoins spécifiques. Il serait donc approprié de promouvoir la mise en œuvre de rencontres citoyennes, inscrites dans des espaces alternatifs, où musulmans et non-musulmans puissent, en toute équité, exprimer leurs attentes respectives vis-à-vis d'un meilleur « vivre-ensemble ».

54. Le rapport d'Unia (2015) démontre qu'entre 2010 et 2015, il y a eu une augmentation de dossiers ouverts pour des raisons de discriminations ou délits de haine en raison de motifs religieux ou philosophiques, parmi lesquels $93 \%$ concernent des personnes de confession musulmane. Le critère religieux, par ailleurs, est le troisième le plus représenté pour des causes de discriminations, après l'âge et le handicap. 55. Célestine Bocquet, Brigitte Maréchal, Sofie Van Den Abeele, Musulmans et non-musulmans en Belgique. Des pratiques prometteuses favorisent le vivre-ensemble, Bruxelles, Fondation Roi Baudouin, 2015. 56. Nous pouvons y insérer les approches d'obédience qui, bien que parallèles à celles socio-éducatives, participent à une meilleure compréhension des croyances et des religions dans notre société sécularisée. 57. Julie Ringelheim rappelle, à l'occasion d'une rencontre sur la lutte contre les discriminations durant les activités « Au fil de l'Autre. De l'identité à l'universalité ", organisées par la Ligue des droits de l'homme, que le système pénal amène à ce que peu de plaintes soient déposées et valorise, en conséquence, les solutions négociées. 